\title{
Nano- and mesoscale structures of amorphous calcium carbonate indicate nanoscale assembly processes
}

\author{
Simon M. Clark, *,\$\$ Bruno Colas,,$\$$ Dorrit E. J acob," J oerg C. Neuefeind, \& Hsiu-Wen Wang, ${ }^{\&}$ \\ Katherine L. Page, \& Alan K. Soper, $\neq$ Philipp I. Schodder, ${ }^{\wedge}$ Patrick Duchstein, \\ Benjamin Apeleo Zubiri, ${ }^{\Delta}$ Tadahiro Yokosawa, ${ }^{\Delta}$ Vitaliy Pipich," Dirk Zahn," Erdmann \\ Spiecker, $\Delta$ and Stephan E. Wolf. $*, \Delta$
}

${ }^{\S}$ School of Engineering, Macquarie University, North Ryde, NSW 2009, Australia.

${ }^{\$}$ Australian Centre for Neutron Scattering, Australian Nuclear Science and Technology Organisation, Locked Bag 2001, Kirrawee DC, NSW 2232, Australia.

\#Research School of Earth Sciences, The Australian National University, Canberra ACT 2600, Australia

«Spallation Neutron Source, Oak Ridge National Laboratory, Pak Ridge, TN 37831, USA.

fISIS Facility, Rutherford Appleton Laboratory, Chilton, Didcot, Oxon, OX11 0QX, UK.

$\triangle$ Department of Materials Science and Engineering (WW), Institute of Glass and Ceramics (WW3), Friedrich-Alexander-University Erlangen-Nuremberg (FAU), Martensstrasse 5, 91058 Erlangen, Germany.

"Department of Chemistry and Pharmacy, Chair for Theoretical Chemistry / Computer Chemistry Centre (CCC), Friedrich-Alexander-University Erlangen-Nuremberg (FAU), Nägelsbachstrasse 25, 91058 Erlangen, Germany.

$\triangle$ Institute of Micro- and Nanostructure Research (IMN) \& Center for Nanoanalysis and Electron Microscopy (CENEM),

Interdisciplinary Center for Nanostructured Films (IZNF), Friedrich-Alexander-University Erlangen-Nuremberg (FAU), Cauerstraße 3, 91058 Erlangen, Germany.

"J ülich Centre for Neutron Science (J CNS), Forschungszentrum J ülich GmbH, Outstation at FRM II, Lichtenbergstrasse 1, 85747 Garching, Germany

$\triangle$ Interdisciplinary Center for Functional Particle Systems (FPS), Friedrich-Alexander University Erlangen-Nürnberg (FAU), Haberstrasse 9a, 91058 Erlangen, Germany

\begin{abstract}
Transient amorphous calcium carbonate (ACC) plays a crucial role as a precursor phase in a range of mineralization systems. Knowledge of the atomic structures of such precursors is essential for elucidating formation pathways and identifying molecular control mechanisms of additives on the crystalline end product. How ever, the challenge of characterising intermediate-range order at high accuracy has impeded the development of a consolidated ultrastructural model for ACC. Here, we approach this issue by applying a method for determining atomically resolved structures of amorphous materials using Monte Carlo simulations constrained by both X-ray and neutron scattering data. This structural analysis approach allows us to develop a detailed model for ACC at the atomic level. Our findings reveal that synthetic $\mathrm{ACC}$, rapidly precipitated at high $\mathrm{pH}$, consists of two-nanometer sized units containing a high degree of near range order similar to partially disordered nano-crystals. Small-angle scattering analyses show a multi-scale hierarchical organisation of the structure, supportive of a multi-step colloid self-assembly process. Computer simulations and high-resolution transmission electron microscopy show that the mesostructure of ACC resembles that of a glassy gel with crystalline material in domains. Our findings support the formation of ACC by a nanoparticle aggregation process that likely starts from prenucleation clusters in solution.
\end{abstract}

[2004 of $\mathbf{2 2 0 0}$ words] A molecular understanding of the formation of amorphous phases is fundamental for controlling crystallization in synthetic and industrial settings, ${ }^{1-3}$ when deciphering biomineralization processes, ${ }^{4-}$ ${ }^{8}$ and the action of additives. ${ }^{9-12}$ Despite its broad relevance as a transient precursor in many natural and synthetic mineralization processes, ${ }^{1-6}$ the molecular structure of amorphous calcium carbonate (ACC) and its molecular formation pathway are a subject of ongoing debate.

Typically for amorphous systems, ACC exhibits polyamorphism with its near-range order depending on the synthesis conditions..$^{7,13-15}$ Its polyamorphism suggests a link of the processes of ACC formation with calcium carbonate prenucleation clusters. ${ }^{7,16-18}$ These are stable solutes, only a few nanometers in size ${ }^{16,17,19,20}$ which form spontaneously in calcium carbonate solutions ${ }^{21}$ based on ion-association equilibria ${ }^{17}$ which are sensitive to conditions in the solution. ${ }^{20}$

Notably, the variable stability of calcium carbonate prenucleation clusters in solution correlates with the solubility of the resulting ACC polyamorph. ${ }^{7}$ This correlation lends support to a formation scenario in which prenucleation clusters serve as a precursory stage of solid ACC, ${ }^{16-}$ ${ }^{18,22}$ a model which was also pushed forward by cryo- 
transmission electron microscopy studies on the early stages of template-mediated calcium carbonate formation. ${ }^{16}$ To date, it is still debated if ACC forms directly from these prenucleation clusters, mainly because consolidated ultrastructural information on bulk ACC has not been available.

Three recent studies of synthetic and non-deuterated ACC using Monte Carlo simulations ${ }^{23}$ constrained by scattering data produced disparate pictures of the ACC structure. The first study, using ACC precipitated without additives, yielded a structural model of a nanoporous charge-separated calcium-rich framework pervaded by interconnected channels rich in water and carbonate. ${ }^{24}$ In contrast, two other studies using $\mathrm{Mg}^{2}$-doped ${ }^{25}$ and nondoped $\mathrm{ACC}^{26}$ proposed a homogenous structure, thus challenging the existence of long-range hydrogenbonded networks.

To evaluate these conflicting models and to obtain a detailed structural model of ACC at the atomic level, we performed neutron and X-ray scattering experiments, combined with Monte Carlo simulations. We explored the model's dynamic properties via molecular simulations and extended our findings to the mesostructure through high-resolution transmission electron microscopy and small/very-small angle neutron scattering.

Synthesis of a deuterated, non-doped ACC stable for many days ${ }^{27}$ enabled us to collect neutron and X-ray scattering data of excellent quality, see Figure S1 provided in the Supplemental Information (SI). Monte Carlo simulations ${ }^{23}$ constrained by these scattering data reveal a highly heterogeneous structure on the mesoscale, composed of anhydrous crystalline nanodomains of around $2 \mathrm{~nm}$, and an interstitial net-like matrix of water molecules, shown in Figure 1. This model agrees with recent stereochemical descriptions based on ${ }^{1} \mathrm{H}$ - and ${ }^{13} \mathrm{C}$ NMR studies evidencing differing water environments in hydrated ACC. ${ }^{28-30}$ Our simulations yield sets of atomic coordinates that allowed us to extract pair-distribution functions, coordination numbers, and bond and torsion angle probability distributions (Fig. S2 - S4 in the SI). These probability functions feature sharp peaks that are characteristic of a high degree of short-range order within the nanodomains indicative of a crystalline nature of these domains (see SI for an extended discussion). This near-range order allowed us to construct an average atomic structure for these anhydrous regions (Fig. S5) which is in good agreement with previous experimental findings. ${ }^{24-26}$

Molecular dynamics studies based on this new nanodomainal model revealed that the nanosecond-dynamics of the ACC are more similar to those of glasses than of solids, see Figure $\mathbf{2}$ and Fig. S6. Nearest-neighbour analyses show hydrate water bound to nanometresized domains. Water molecules and, to a lesser extent, carbonate ions display orientational flexibility. Nanodomain deformation and ion migration in the ACC allow for viscous mobility with diffusion constants that are four to five orders of magnitude lower than those of aqueous solutions. Subsequent simulations showed that, on average, only five carbonate ions and no $\mathrm{Ca}$ ions dissociate from the ACC hydrate surface exposed to a
$7.5 \mathrm{~nm}$-sized film of water molecules, demonstrating the robustness of our modelled nano-structure against dissolution. While we observed a dynamic equilibrium of carbonate dissociation and re-association in 10 ns runs, no calcium was released in a model with a surface area of $37 \mathrm{~nm}^{2}\left(43 \times 43 \times 159 \AA^{3}\right)$. This result validates the chemical stability of our structural model.

High-resolution transmission electron microscopy (HRTEM) and selected area electron diffraction (SAED) corroborated the existence of the nanodomains, see Figure 3. The bright-field TEM (BF-TEM) image in Fig. 3a shows the nanoparticles, approximately $50 \mathrm{~nm}$ in size, that make up the ACC hydrate and are interconnected via necking. Their SAED patterns display diffuse rings at $3.549 \mathrm{~nm}^{-1}$ and $5.315 \mathrm{~nm}^{-1}$ (inset in Fig. 3a). Diffuse diffraction rings are generally accepted as evidence for amorphicity, with the variability of the ring positions attributed to polyamorphism $m^{6,7,16,31}$, but can be equally indicative for nano-crystalline materials. ${ }^{31,32}$ Given that the nanodomains feature a strong near-range order, we conducted Scherrer analysis of the first ring in the SAED pattern and received a mean diameter of the nanodomains of $1.8 \mathrm{~nm}$, a value which agrees well with the two-nanometre size for the nanodomains as determined by scattering experiments and Monte-Carlo-simulations. Likewise, the measured peak positions in the SAED patterns in reciprocal space (inset in Fig. 3a) correspond to mean interatomic distances of $0.28 \mathrm{~nm}$ and $0.19 \mathrm{~nm}$, respectively. These interatomic distances are very similar to those derived from our $X$-ray and neutron scattering data (Fig. S2) as well as to those in recently published X-ray absorption spectroscopy studies ${ }^{7}$.

In addition to HRTEM and SAED, we carried out a controlled in situ crystallization experiment in the TEM by stepwise increasing the radiation dose with a widespread electron beam in TEM mode. During this experiment we observed that the first crystalline phases forming were nanodomains with a lateral diameter of about two nanometres (Fig. $3 \mathrm{~b}$ ) which correspond well with the sizes in our structural model. These nanodomains show a lattice spacing of $0.24 \mathrm{~nm}$ that fits the $\mathrm{Ca}-\mathrm{O}$ distances in aragonite $\left(0.2416 \mathrm{~nm}, \mathrm{~d}_{031}\right.$ aragonite $=0.2410 \mathrm{~nm}$; ICSD no. 32100 ), and that also matches the $\mathrm{Ca}-\mathrm{O}$ distances in calcium oxide $\left(\mathrm{d}_{200}{ }^{\mathrm{CaO}}=0.2408 \mathrm{~nm}\right.$; ICSD 51409). C CaO could have formed resulting from beam damage which we tried to avoid, but cannot completely rule out. The observed lattice spacing also agrees well with the $\mathrm{Ca}-\mathrm{O}$ distances determined for ACC in our scattering experiments (Fig. S2). Further electron beam irradiation saw the formation of larger crystalline areas of up to $30 \mathrm{~nm}$, while patchy areas of amorphous material remained, see top part of Fig. 3c, d. The lattice spacings of these newly developed, larger crystalline regions are indicative of calcite (see Fig. 3c) and of either aragonite or vaterite (see Fig. $3 d$ ) but not of calcium oxide.

Neutron scattering experiments in both the small and very-small angle regime (V/SANS) revealed a four-levelled hierarchical structure of ACC, see Figure 4. The scattering data shows four individual Guinier regimes evidencing four independent structural levels (Fig. S7). The 
smallest hierarchical unit detected by V/SANS has a radius of about $3 \mathrm{~nm}$, which is similar in size to the fundamental nanodomains $(2 \mathrm{~nm})$ in our ACC model. These fundamental units aggregate, separated by chains of water molecules to form a second hierarchical level with a typical size of $55 \mathrm{~nm}$ (40-60 nm in TEM analysis) (Fig. 3a). The third and fourth hierarchical levels are in the size regimes of $370 \mathrm{~nm}$ and $2.8 \mu \mathrm{m}$, respectively.

Taking all results together shows an ACC structure consisting of anhydrous calcium carbonate nanodomains of ca. $2 \mathrm{~nm}$ diameter as the fundamental unit. The nanodomains are characterised by their high degree of order, a finding which is supportive of the nanocrystalline model for ACC of Rez al. ${ }^{31}$ Aggregation of these nanoparticles yields a hierarchically-organised glassy gel with chains of water molecules separating the nanodomains. This model is consistent with results on ACC synthesized in the presence of polymers and other capping agents, which showed stabilization of individual two-nanometer large nanoparticles of amorphous calcium carbonate with distinct near-range order. ${ }^{33,34}$ It also fits cryo-transmission electron microscopy studies on the very early stages of heterogenous calcium carbonate formation. ${ }^{16}$

The multi-level hierarchically-organized ACC structure in a single-component precipitate can be rationalized via a multi-step complex particle attachment process, similarly to some observed in other self-assembly systems..$^{35}$ Our findings suggest that the fundamental $2 \mathrm{~nm}$-sized units are solution-borne entities whose formation is triggered by the increasing activity product in the solution.
Such spontaneously-forming nanoscaled solute species, termed prenucleation clusters, have been shown to exist in the calcium carbonate system and have diameters of 1 to 4 nanometres. . $^{16,17,19,20}$

We envisage ACC formation to be driven by a multiscale particle aggregation and coalescence process which starts from discreet and well-ordered fundamental nano-units derived from prenucleation clusters. Hence, our study provides a mechanistic link between the prenucleation clusters in solution and their transformation into the solid-state phase. The hierarchical organization of the ACC structure is likely facilitating the control-mechanisms for polyelectrolytes, such as biomineralization proteins ${ }^{5,6}$ or precipitation inhibitors ${ }^{36}$ across multiple length scales.

The results presented here pushes our current understanding of the formation of amorphous mineral phases further towards a particle self-assembly process starting from complex solute species, and correlates with recent observations in other inorganic systems. ${ }^{37}$ The novel combination of methods allows for exploring the mesoand ultrastructure of ACC in response to specific synthesis conditions and the impact of reaction conditions on the chemical and isotopic composition of biominerals ${ }^{8}$. This unprecedented level of comprehensive ultrastructural information enables more accurate structural modelling of the major interaction partners and better mechanistic insight into the genesis of amorphous and crystalline solid-state materials from solutions.

\section{Author Contributions}

SC, DEJ and SEW designed the study. HWW, KP, and PIS devised the sample synthesis method and made the sample. J N collected and processed the neutron data. AKS carried out the EPSR refinement; PD conducted the molecular modelling simulations. VP conducted V/SANS experiments; VP and SEW analysed the V/SANS data. BAZ, TY and PIS performed TEM analyses; BAZ, TY, PIS, ES, and SEW interpreted and analysed the results. SMC, BC, DEJ , SEW and DZ interpreted the results of the simulations, built the atomic model, and wrote the manuscript with contributions from all authors.

\section{Funding Sources}

BC was supported by a PhD scholarship provided jointly by ACNS, ANSTO and Macquarie University. HWW, KP and J N was supported by the US Department of Energy (DOE), Office of Science (SC), Office of Basic Energy Sciences (BES), Chemical Sciences, Geosciences, and Biosciences Division. KP was supported in part by the DOE, SC, BES Early Career Research Program (KC 040602), under contract number DE-AC 05-00OR22725. The NOMAD instrument at ORNL's SNS is sponsored by the DOE, SC, BES, Scientific User Facilities Division. The instrument 11-ID-B was supported by the DOE, SC, BES, under Contract No. DE-AC02-06CH11357. DEJ is supported by the Australian Research Council (FT120100462, DP 160102081). SEW acknowledges support by an Emmy Noether starting grant issued by the German Research Foundation (Deutsche Forschungsgemeinschaft DFG, grant number 251939425), by a BayIntAn grant by the Bavarian Research Alliance (FAU_2018_07); SEW also acknowledges financial support by J CNS for neutron scattering measurements at the Heinz Maier-Leibnitz Zentrum (MLZ), Garching, Germany. SEW, BAZ, TY, and ES acknowledge support from the Cluster of Excellence 315 'Engineering of Advanced Materials - Hierarchical Structure Formation for Functional Devices' funded by the German Research Foundation. BAZ, TY, and ES acknowledge funding by the German Research Foundation - Project-ID 416229255 - SFB 1411 
a

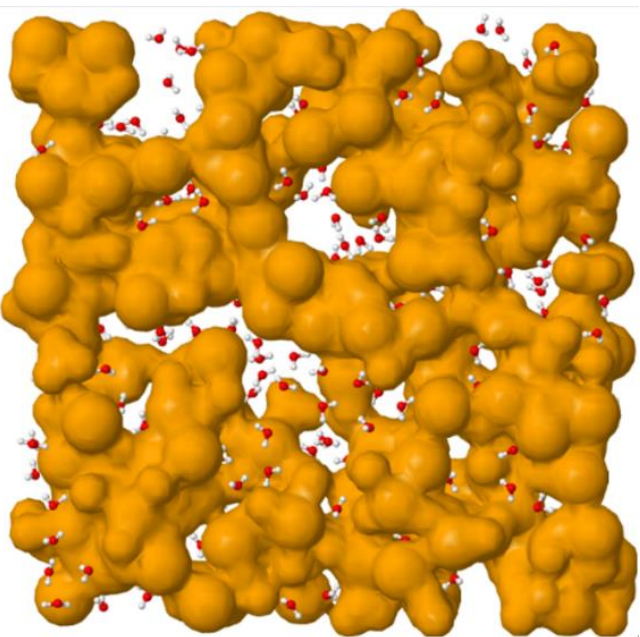

b

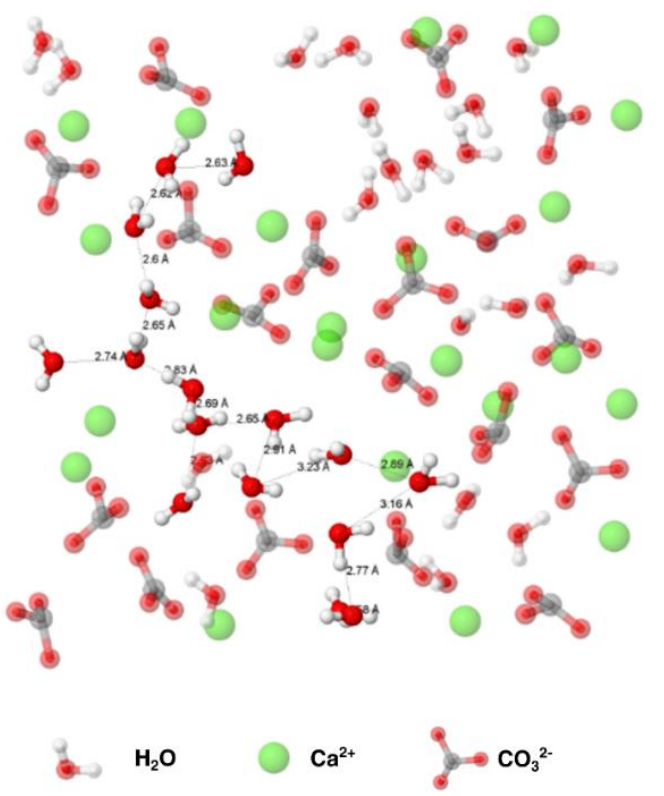

Figure 1. Projections of the $A C C$ structure model produced by Monte Carlo simulations. (a) Calcium carbonate units form anhydrous clusters; water is distributed around these clusters with no substantive regions of bulk water. Calcium atoms are represented by oversized yellow coloured spheres and water by a ball-and-stick models; the carbonates are contained within the oversized spheres. (b) Expanded view of subfigure a. Water molecules form chains around anhydrous calcium carbonate clusters, a highlighted one demonstrates a typical arrangement with a periodically branching chain. The oxygen-oxygen intermolecular distances are shown along the chain, and the hydrogen-hydrogen distance in one water molecule is about $1.5 \AA$ a

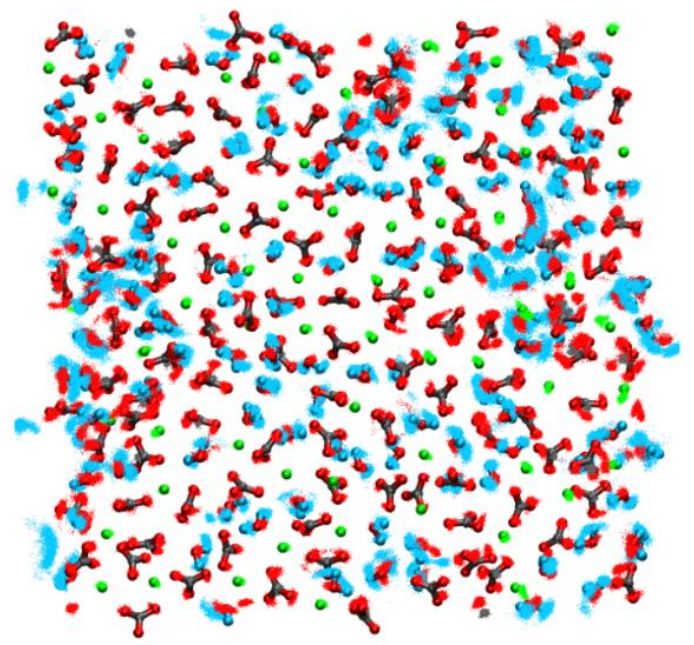

b

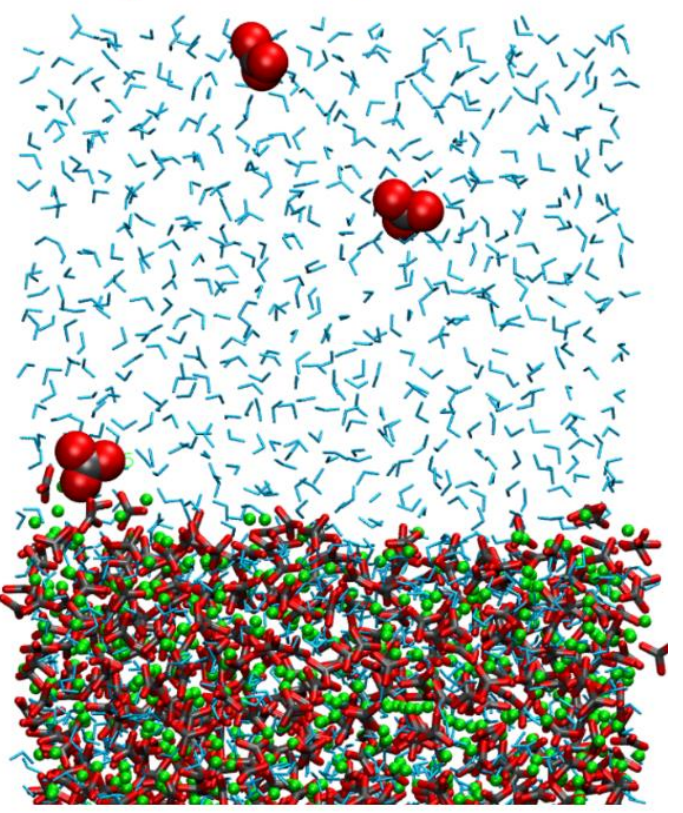

Figure 2. Molecular dynamics simulations on the structural stability of the nanodomainal model (Ca: green, O: red, C: grey, $\mathrm{H}$ : blue). (a) Cross-section through a cubic, periodic box of the ACC model with an edge length of $42.97 \AA$ containing $1000 \mathrm{CaCO}_{3}$ and $1200 \mathrm{H}_{2} \mathrm{O}$. The light-blue point clouds represent atom positions within a timeframe of 2 ns. The structure is best described in terms of a glassy calcium carbonate as ion positions are rather rigid while water diffuses through the structure. (b) Projection of the model in contact with a slab of water - the viewing direction is along the ACC surface. Only carbonate ions are continuously released and re-adsorbed leading to an dynamical interfacial equilibrium. 
a

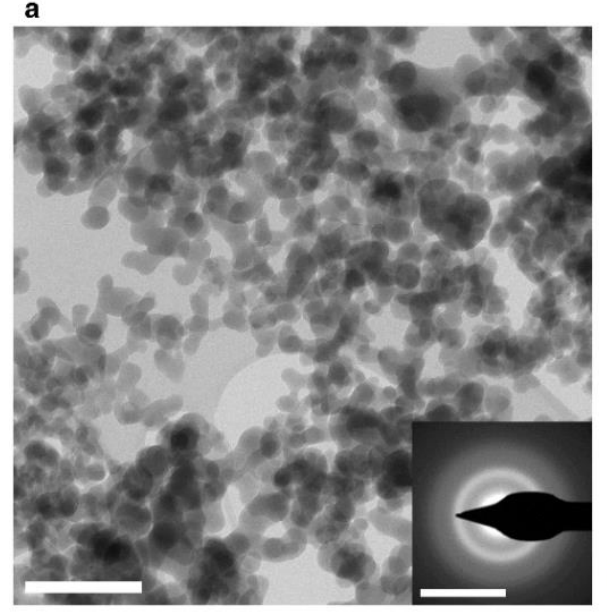

b
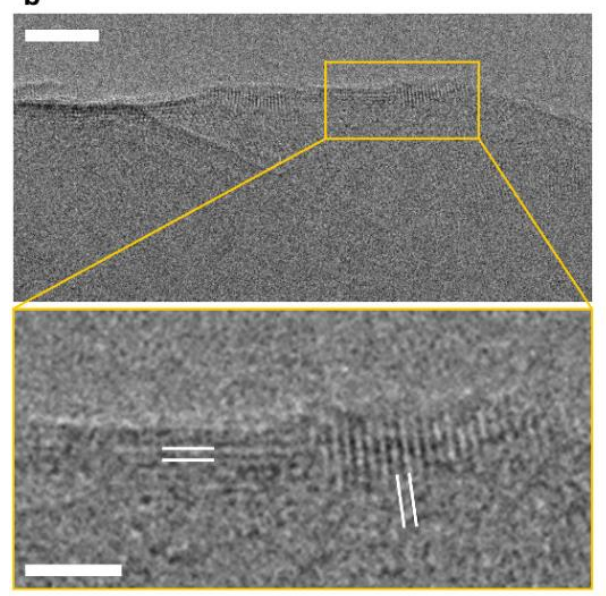

c
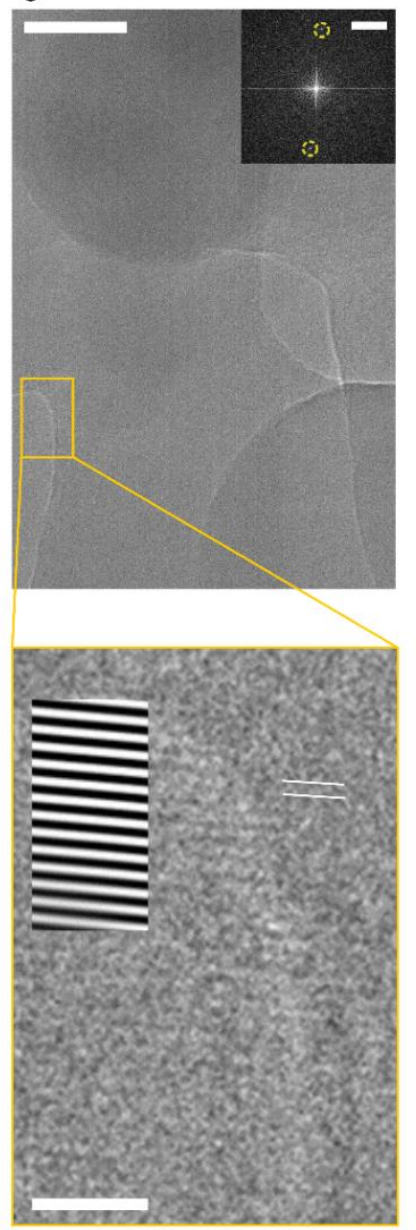

d
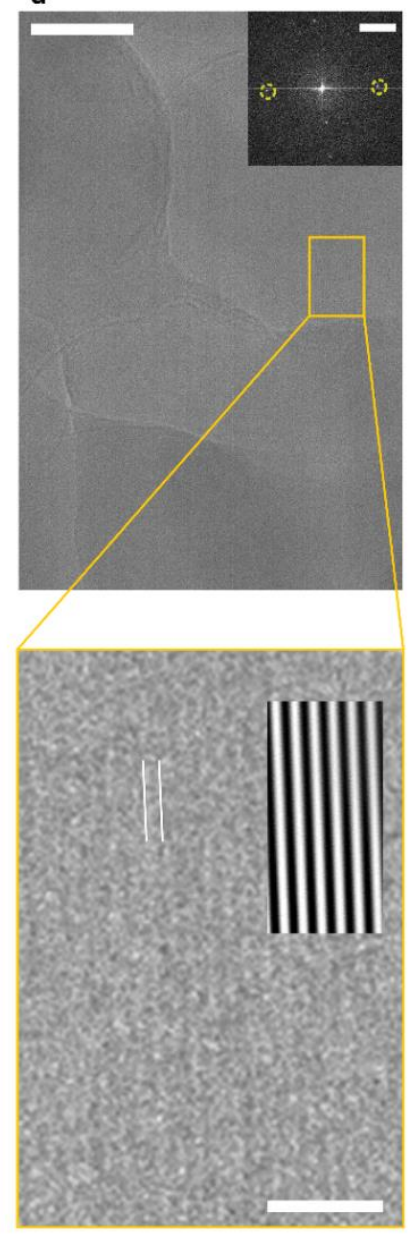

Figure 3. TEM, SAED, and FFT analyses of the synthesized ACC. (a) Bright-field TEM overview of ACC dispersed on a Lacey carbon support grid. The inset shows the SAED pattern with diffuse rings at $3.549 \mathrm{~nm}^{-1}$ and $5.315 \mathrm{~nm}^{-1}$. (b) HRTEM micrographs of an edge region of the ACC nanostructure, irradiated with as little electron dose as possible, showing crystalline regions with a few $\mathrm{nm}$ in size with a lattice spacing of $0.24 \mathrm{~nm}$, as indicated with white lines. (c-d) HRTEM images after further controlled electron beam irradiation: larger crystalline regions of $10-30 \mathrm{~nm}$ form which give pronounced signals in the FFT spectra (inset). The lower micrographs are enlarged views of the regions marked above. FFT-filtered areas highlight the contrast variation of the observed lattice fringes (banded overlays), when using the yellow-marked signals in the upper FFT spectra. The observed lattice spacings of $0.303 \mathrm{~nm}$ observed in subfigure $c$ and of $0.325 \mathrm{~nm}$ observed in subfigure $\mathrm{d}$ coincide with those of calcite and of either vaterite or aragonite $\left(d_{104}{ }^{\text {calcite }}=0.3024 \mathrm{~nm}\right.$, ICSD 18164; $d_{113}{ }^{\text {vaterite }}=0.3282 \mathrm{~nm}$, AMCSD 0019870; $\mathrm{d}_{021}$ aragonite $=0.3273 \mathrm{~nm}$; ICSD 32100).

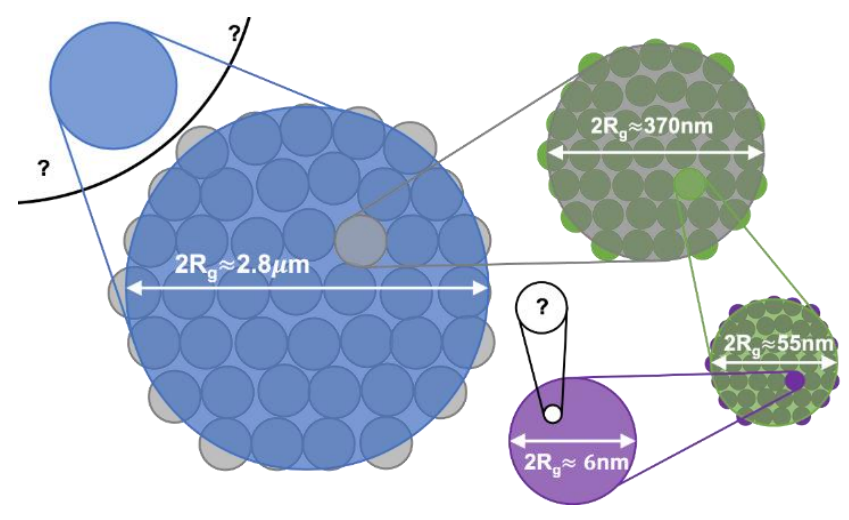

Figure 4. Scheme of the hierarchical organization of ACC precipitated at high $\mathrm{pH}$ as revealed by neutron scattering analyses in the small- to very-small-angle regime. 


\section{REFERENCES}

(1) De Yoreo, J . J .; Gilbert, P. U. P. A.; Sommerdijk, N. A. J. M. J . M.; Penn, R. L.; Whitelam, S.; J oester, D.; Zhang, H.; Rimer, J. D.; Navrotsky, A.; Banfield, J. F.; et al. Crystallization by Particle Attachment in Synthetic, Biogenic, and Geologic Environments. Science 2015, 349 (6247), aaa6760-aaa6760.

Natalio, F.; Corrales, T. P.; Panthofer, M.; Schollmeyer, D.; Lieberwirth, I.; Muller, W. E. G.; Kappl, M.; Butt, H.-J.; Tremel, W. Flexible Minerals: Self-Assembled Calcite Spicules with Extreme Bending Strength. Science 2013, 339 (6125), 1298-1302. https://doi.org/10.1126/science.1216260.

(3) Gower, L. B. Biomimetic Model Systems for Investigating the Amorphous Precursor Pathway and Its Role in Biomineralization. Chem. Rev. 2008, 108 (11), 4551-4627. https://doi.org/10.1021/cr800443h.

(4) Von Euw, S.; Zhang, Q.; Manichev, V.; Murali, N.; Gross, J.; Feldman, L. C.; Gustafsson, T.; Flach, C.; Mendelsohn, R.; Falkowski, P. G. Biological Control of Aragonite Formation in Stony Corals. Science 2017, 356 (6341), 933-938. https://doi.org/10.1126/science.aam6371.

(5) Weiner, S.; Addadi, L. Crystallization Pathways in Biomineralization. Annu. Rev. Mater. Res. 2011, 41 (1), $21-$ 40. https://doi.org/10.1146/annurev-matsci-062910095803.

(6) Bahn, S. Y.; J O, B. H.; Choi, Y. S.; Cha, H. J. Control of Nacre Biomineralization by Pif80 in Pearl Oyster. Sci. Adv. 2017, 3 (8). https://doi.org/10.1126/sciadv.1700765.

(7) Cartwright, J. H. E.; Checa, A. G.; Gale, J. D.; Gebauer, D.; Sainz-Díaz, C. I. Calcium Carbonate Polyamorphism and Its Role in Biomineralization: How Many Amorphous Calcium Carbonates Are There? Angew. Chem. Int. Ed. 2012, 51 (48), 11960-11970. https://doi.org/10.1002/anie.201203125.

(8) Jacob, D. E.; Wirth, R.; Agbaje, O. B. A.; Branson, O.; Eggins, S. M. Planktic Foraminifera Form Their Shells via Metastable Carbonate Phases. Nat. Commun. 2017, 8 (1), 1265. https://doi.org/10.1038/s41467-017-00955-0.

(9) Ihli, J .; Kim, Y.-Y.; Noel, E. H.; Meldrum, F. C. The Effect of Additives on Amorphous Calcium Carbonate ( ACC ): $\mathrm{J}$ anus Behavior in Solution and the Solid State. Adv. Funct. Mater. 2013, 1575-1585. https://doi.org/10.1002/adfm.201201805.

(10) Zou, Z.; Yang, X.; Albéric, M.; Heil, T.; Wang, Q.; Pokroy, B.; Politi, Y.; Bertinetti, L. Additives Control the Stability of Amorphous Calcium Carbonate via Two Different Mechanisms: Surface Adsorption versus Bulk Incorporation. Adv. Funct. Mater. 2020, 30 (23), 2000003. https://doi.org/10.1002/adfm.202000003.

(11) Bots, P.; Benning, L. G.; Rodriguez-Blanco, J . D.; RoncalHerrero, T.; Shaw, S. Mechanistic Insights into the Crystallization of Amorphous Calcium Carbonate (ACC). Cryst. Growth Des. 2012, 12 (7), 3806-3814. https://doi.org/10.1021/cg300676b.

(12) Kababya, S.; Gal, A.; Kahil, K.; Weiner, S.; Addadi, L.; Schmidt, A. Phosphate-Water Interplay Tunes Amorphous Calcium Carbonate Metastability: Spontaneous Phase Separation and Crystallization vs Stabilization Viewed by Solid State NMR. J. Am. Chem. Soc. 2015, 137 (2), 990998. https://doi.org/10.1021/ja511869g.

(13) Farhadi Khouzani, M.; Chevrier, D. M.; Güttlein, P.; Hauser K.; Zhang, P.; Hedin, N.; Gebauer, D. Disordered Amorphous Calcium Carbonate from Direct Precipitation. CrystEngComm 2015, 17 (26), 4842-4849. https://doi.org/10.1039/C5CE00720H.

(14) Gebauer, D.; Gunawidjaja, P. N.; Ko, J. Y. P.; Bacsik, Z. Z.; Aziz, B.; Liu, L.; Hu, Y.; Bergström, L.; Tai, C.-W. W.; Sham, T.-K. K.; et al. Proto-Calcite and Proto-Vaterite in Amorphous Calcium Carbonates. Angew. Chem. Int. Ed.
2010,
122
(47),
9073-9075.

https://doi.org/10.1002/ange.201003220.

(15)
Lam, R. S. K.; Charnock, J. M.; Meldrum, F. C.; Lennie, A.; Meldrum, F. C. Synthesis-Dependant Structural Variations in Amorphous Calcium Carbonate. CrystEngComm 2007, 9 (12), 1226-1236. https://doi.org/10.1039/b710895h.

Pouget, E. M.; Bomans, P. H. H.; Goos, J. A. C. M.; Frederik, P. M.; de With, G.; Sommerdijk, N. A. J. M.; Bomans, P. H. H.; Goos, J. A. C. M.; Frederik, P. M.; de With, G.; et al. The Initial Stages of Template-Controlled $\mathrm{CaCO} 3$ Formation Revealed by Cryo-TEM. Science 2009, 323

https://doi.org/10.1126/science.1169434.

Gebauer, D.; Völkel, A.; Cölfen, H. Stable Prenucleation Calcium Carbonate Clusters. Science 2008, 322 (5909), 1819-1822. https://doi.org/10.1126/science.1164271.

Gebauer, D.; Wolf, S. E. Designing Solid Materials from Their Solute State: A Shift in Paradigms toward a Holistic Approach in Functional Materials Chemistry. J . Am. Chem. Soc. 2019, $141 \quad$ (11), 4490-4504. https://doi.org/10.1021/jacs.8b13231.

Mohammed, A. S. A.; Carino, A.; Testino, A.; Andalibi, M. R.; Cervellino, A. In Situ Liquid SAXS Studies on the Early Stage of Calcium Carbonate Formation. Part. Part. Syst. $\begin{array}{lllll}\text { Charact. } & \mathbf{2 0 1 9} & 36 & (6), & 1800482 .\end{array}$ https://doi.org/10.1002/ppsc.201800482.

Demichelis, R.; Raiteri, P.; Gale, J. D.; Quigley, D.; Gebauer, D. Stable Prenucleation Mineral Clusters Are Liquid-like Ionic Polymers. Nat. Commun. 2011, 2 (590), 590. https://doi.org/10.1038/ncomms1604.

Wallace, A. F.; Hedges, L. O.; Fernandez-martinez, A.; Raiteri, P.; Gale, J. D.; Waychunas, G. A.; Whitelam, S.; Banfield, J. F.; De Yoreo, J.J. Microscopic Evidence for Liquid-Liquid Separation in Supersaturated $\mathrm{CaCO}_{3}$ Solutions. Science 2013, 341 (6148), 885-889. https://doi.org/10.1126/science.1230915.

Gebauer, D.; Cölfen, H. Prenucleation Clusters and NonClassical Nucleation. Nano Today 2011, 6 (6), 564-584. https://doi.org/10.1016/j.nantod.2011.10.005.

Soper, A. K. Empirical Potential Monte Carlo Simulation of Fluid Structure. Chem. Phys. 1996, 202 (2-3), 295-306. https://doi.org/10.1016/0301-0104(95)00357-6.

Goodwin, A. L.; Michel, F. M.; Phillips, B. L.; Keen, D. A.; Dove, M. T.; Reeder, R. J. Nanoporous Structure and Medium-Range Order in Synthetic Amorphous Calcium Carbonate. Chem. Mater. 2010, 22 (10), 3197-3205. https://doi.org/10.1021/cm100294d.

Cobourne, G.; Mountjoy, G.; Rodriguez-Blanco, J. D.; Benning, L. G.; Hannon, A. C.; Plaisier, J . R. Neutron and $X$-Ray Diffraction and Empirical Potential Structure Refinement Modelling of Magnesium Stabilised Amorphous Calcium Carbonate. J. Non. Cryst. Solids 2014, 401, 154-158. https://doi.org/10.1016/j.jnoncrysol.2013.12.023.

J ensen, A. C. S.; Imberti, S.; Parker, S. F.; Schneck, E.; Politi, Y.; Fratzl, P.; Bertinetti, L.; Habraken, W. J. E. M. Hydrogen Bonding in Amorphous Calcium Carbonate and Molecular Reorientation Induced by Dehydration. J. Phys.

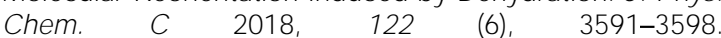
https://doi.org/10.1021/acs.jpcc.7b10459.

Wang, H.-W. W.; Daemen, L. L.; Cheshire, M. C.; Kidder, M. K.; Stack, A. G.; Allard, L. F.; Neuefeind, J .; Olds, D.; Liu, J.; Page, K.; et al. Synthesis and Structure of Synthetically Pure and Deuterated Amorphous (Basic) Calcium Carbonates. Chem. Commun. 2017, 53 (20), 2942-2945. https://doi.org/10.1039/c6cc08848a.

Reeder, R. J .; Tang, Y.; Schmidt, M. P.; Kubista, L. M.; Cowan, D. F.; Phillips, B. L. Characterization of Structure in Biogenic Amorphous Calcium Carbonate: Pair Distribution Function and Nuclear Magnetic Resonance Studies of Lobster Gastrolith. Cryst. Growth Des. 2013, 13 (5), 1905-1914. https://doi.org/10.1021/cg301653s. Sen, S.; Kaseman, D. C.; Colas, B.; J acob, D. E.; Clark, S. $\mathrm{M}$. Hydrogen Bonding Induced Distortion of $\mathrm{CO} 3$ Units and Kinetic Stabilization of Amorphous Calcium Carbonate: 
Results from 2D 13 C NMR Spectroscopy. Phys. Chem. Chem. Phys. 2016, 7, 689-702. https://doi.org/10.1039/C6CP02729F.

(30) Ihli, J.; Wong, W. C.; Noel, E. H.; Kim, Y.-Y.; Kulak, A. N.; Christenson, H. K.; Duer, M.J .; Meldrum, F. C. Dehydration and Crystallization of Amorphous Calcium Carbonate in Solution and in Air. Nat. Commun. 2014, 5, 3169. https://doi.org/10.1038/ncomms4169.

(31) Rez, P.; Sinha, S.; Gal, A. Nanocrystallite Model for Amorphous Calcium Carbonate. J. Appl. Crystallogr. 2014 47 (5), $1651-1657$ https://doi.org/10.1107/S1600576714018202.

(32) Treacy, M. M. J.; Gibson, J. M. Variable Coherence Microscopy: A Rich Source of Structural Information from Disordered Materials. Acta Crystallogr. Sect. A Found. Crystallogr. 1996, $52 \quad$ (2), 212-220. https://doi.org/10.1107/S0108767395012876.

(33) Xu, Y.; Tijssen, K. C. H.; Bomans, P. H. H.; Akiva, A.; Friedrich, H.; Kentgens, A. P. M.; Sommerdijk, N. A. J. M Microscopic Structure of the Polymer-Induced Liquid Precursor for Calcium Carbonate. Nat. Commun. 2018, 9

(1), 1-12. https://doi.org/10.1038/s41467-018-05006-w.

(34) Sun, S.; Chevrier, D. M.; Zhang, P.; Gebauer, D.; Cölfen, H Distinct Short-Range Order is Inherent to Small Amorphous Calcium Carbonate Clusters $(<2 \mathrm{Nm})$. Angew. Chem. Int. Ed. 2016, 55 (40), 12206-12209. https://doi.org/10.1002/anie.201604179.

(35) Zeng, C.; Chen, Y.; Kirschbaum, K.; Lambright, K. J .; J in R. Emergence of Hierarchical Structural Complexities in Nanoparticles and Their Assembly. Science 2016, 354 (6319),

$1580-1584$ https://doi.org/10.1126/science.aak9750.

(36) Hasson, D.; Shemer, H.; Sher, A. State of the Art of Friendly "Green" Scale Control Inhibitors: A Review Article. Ind. Eng. Chem. Res. 2011, 50 (12), 7601-7607. https://doi.org/10.1021/ie200370v.

(37) Lukić, M. J.; Wiedenbeck, E.; Reiner, H.; Gebauer, D. Chemical Trigger toward Phase Separation in the Aqueous Al(III) System Revealed. Sci. Adv. 2020, 6 (23), 1-8. https://doi.org/10.1126/sciadv.aba6878. 